\title{
Formulation and Evaluation of Jamun RTS Blended with Avocado and Nannari - A Nutraceutical Drink
}

\author{
Arshad Khayum ${ }^{1 *}$, H.C. Krishna ${ }^{1}$, A.P. Mallikarjuna Gowda ${ }^{2}$, \\ G.K. Sadananda ${ }^{1}$ and T.H. Shankarappa ${ }^{1}$ \\ ${ }^{1}$ College of Horticulture, University of Horticultural Sciences Campus, GKVK Post, \\ Bengaluru-560065, Karnataka, India \\ ${ }^{2}$ ICAR-KVK, Doddaballapur, Bengaluru Rural-561 205, Karnataka, India
}

*Corresponding author

\section{A B S T R A C T}

Keywords

RTS, Jamun, avocado, Nannari, Honey, Nutraceutical

Article Info

Accepted: 04 May 2018 Available Online: 10 June 2018
Formulation of Jamun RTS, a nutraceutical beverage by blending Jamun with avocado and nannari, was conducted at College of Horticulture, Bangalore. The prepared Jamun RTS was analysed for physico-chemical characters and sensory quality at monthly interval for three months of storage. The TSS, $\mathrm{pH}$, total sugars and reducing sugars were found to have increased during the storage whereas; acidity, anthocyanin, ascorbic acid content, total antioxidants, non- reducing sugars and the organoleptic scores had decreased. Among all the blended RTS treatments, highest $\mathrm{pH} 4.50$ was observed in treatment, $10 \mathrm{ml}$ juice containing 65: 30: 05 percents of jamun, avocado and nannari and sugar. While, maximum TSS $\left(17.60{ }^{\circ} \mathrm{B}\right)$, ascorbic acid $\left(24.72 \mathrm{mg} 100 \mathrm{~g}^{-1}\right)$, higher retention of anthocyanin content $\left(11.80 \mathrm{mg} 100 \mathrm{ml}^{-1}\right)$, total antioxidants $(56.00 \%)$ and total sugars $(13.42 \%)$ was found in treatment, $16 \mathrm{ml}$ juice containing 80: 15: 05 percents of jamun, avocado and nannari and honey.

\section{Introduction}

Adequate knowledge in post-harvest handling, quality assurance and standardization are the important constraints in developing new value added products. Value addition is one of the approaches to enhance the nutraceutical value of a beverage. The beverage of nutraceutical value is prepared by blending two or more plant sources namely fruits, vegetables and seeds, roots, stems and barks of medicinal plants etc. in different combinations and ratios. Ravindra et al., (2012) reported that two or more fruit juices or pulps may be blended in various proportions for the preparation of more palatable and nutritious nectar and ready-to-serve (RTS) beverages. The beverages are also enriched with natural components extracted from the raw sources such as sugars, vitamins, minerals and other biologically active compounds that enhance the health of consumer (Hamid salari et al., 2012).

In the present investigation we have tried the formulation and standardization of jamun blended RTS so as to provide maximum nutritional value to the product. Wherein, 
jamun (Syzygium cuminii L.) fruit has been valued for possessing variety of therapeutic properties. Jamun fruit is rich in carbohydrates, vitamins and minerals such as manganese, zinc, iron, calcium, sodium and potassium. The edible pulp contains various phytochemicals like vitamin C, vitamin A, riboflavin, choline, anthocyanins and various other polyphenols. (Kapoor and Ranote, 2015).

Jamun fruit contains 83.7-85.8\% moisture, 0.7-0.129 g protein, $0.15-0.3 \mathrm{~g}$ fat, 0.3-0.9 g crude fibre, $14 \mathrm{~g}$ carbohydrate, $0.32-0.4 \mathrm{~g}$ ash, 8.3-15 mg calcium, 35mg magnesium, 15-16.2 $\mathrm{mg}$ phosphorus, $1.2-1.62 \mathrm{mg}$ iron, $26.2 \mathrm{mg}$ sodium, $55 \mathrm{mg}$ potassium, $0.23 \mathrm{mg}$ copper, 13 $\mathrm{mg}$ sulphur, $8 \mathrm{mg}$ chlorine, $0.008-0.03 \mathrm{mg}$ thiamine, 0.009- $0.01 \mathrm{mg}$ riboflavin, 0.2-0.29 $\mathrm{mg}$ niacin, 5.7-18 $\mathrm{mg}$ ascorbic acid and $3 \mu \mathrm{g}$ folic acid $100 \mathrm{~g}^{-1}$ of edible portion (Swamy et al., 2012).

Avocado or butter fruit (Persea americana L.) is reputed as nourishing food for diabetic patients due to its low sugar content. Avocado is eaten fresh with bread or in salads, but in India, people prefer to eat it after mixing the pulp with sugar (Chadha, 2001). And the important medicinal crop, swallow root (Decalepis hamiltonii Wight \& Arn.) commonly called as nannari or makaliberu in Kannada, is known to stimulate appetite, relive flatulence, demulcent, diaphoretic, diuretic and as a general tonic (Nayar et al., 1978). It is useful in the treatment of fever, skin disease, diarrhoea, nutrition disorders, blood purifier and has a characteristic flavouring principle (Anon, 1990). Mainly, Jamun fruits are processed to make jam, jellies, squash, vinegar and ice cream for its pleasing and attractive purple colour due to presence of pigments anthocyanin. Thus, flavonoids present in the fruit play dual role as attractive natural colorants which also add nutrition to the beverage and helps improve overall quality of beverage. Therefore, development of value added product from Jamun blended with avocado and nannari could find importance as health drink.

\section{Materials and Methods}

The Jamun fruits, avocado and swallow root (nannari) were procured from the local market, sorted to select ripe, disease and pest free, healthy and undamaged fruits and washed to remove any foreign matter and juices were extracted as per the procedure (Srivastava and Kumar, 1994). Five fruits were selected randomly and tested for physical parameters such as fruit weight and juice recovery percentage. The values were averaged to record the observations. For fruit weights, the fruits were weighed in an electronic weighing balance and the values were recorded in kilograms. The Juice recovery was calculated by dividing volume of fruit juice recovered $(\mathrm{ml})$ with total fruit weight used (g) and expressed in per cent. Jamun fruits was pulped by hand pressing, seeds were removed and the pulp was blended using blender, strained and the juice was collected and the value was measured. Avocado pulp was obtained by cut open the fruit, seeds were removed and pulp was blended using blender. The pulp was collected and value was measured. The average fruit length, diameter and weight of Jamun and avocado fruit were found to be 2.65 and 9.50 $\mathrm{cm}, 1.65$ and $6.96 \mathrm{~cm}, 20$ and $152 \mathrm{~g}$ respectively. The Jamun fruit is constituted by 74 per cent pulp and 26 per cent seed whereas, the avocado fruit contained 66 per cent pulp and 34 per cent seed (Table 1).

The fruit juices or pulp prepared were blended in different juice percentages such as $10 \mathrm{ml}$, $13 \mathrm{ml}$ and $16 \mathrm{ml}$ with different ratios viz., 65:30:05 and 80:15:05 of Jamun, avocado and nannari by using either cane sugar or honey as per the respective treatment combinations. 
Totally there were 12 treatments and they were replicated three times, juice contents (10 $\mathrm{ml}, 13 \mathrm{ml}$ and $16 \mathrm{ml}$ ); juice proportions (65:30:05 and 80:15:05) and sugar source (Cane sugar and honey). The final volume of individual treatment was made up to $200 \mathrm{ml}$ by addition of sugar syrup or honey to have TSS $15^{\circ}$ Brix and acidity to 0.3 percent. Sodium benzoate at $70 \mathrm{ppm}$ was used as preservative. RTS beverages were stored in $200 \mathrm{ml}$ capacity glass bottles at ambient conditions and studied for their nutritional and keeping quality up to three months.

Following are the treatment formulations studied for jamun RTS,

$\mathrm{T}_{1}\left(\mathrm{~J}_{1} \mathrm{~S}_{1} \mathrm{R}_{1}\right): 10 \mathrm{ml}$ juice, (65\% Jamun: $30 \%$ avocado: $5 \%$ nannari), cane sugar

$\mathrm{T}_{2}\left(\mathrm{~J}_{2} \mathrm{~S}_{1} \mathrm{R}_{1}\right): 13 \mathrm{ml}$ juice, (65\% Jamun: $30 \%$ avocado: $5 \%$ nannari), cane sugar

$\mathrm{T}_{3}\left(\mathrm{~J}_{3} \mathrm{~S}_{1} \mathrm{R}_{1}\right): 16 \mathrm{ml}$ juice, (65\% Jamun: $30 \%$ avocado: $5 \%$ nannari), cane sugar

$\mathrm{T}_{4}\left(\mathrm{~J}_{1} \mathrm{~S}_{1} \mathrm{R}_{2}\right): 10 \mathrm{ml}$ juice, (80\% Jamun: $15 \%$ avocado: $5 \%$ nannari), cane sugar

$\mathrm{T}_{5}\left(\mathrm{~J}_{2} \mathrm{~S}_{1} \mathrm{R}_{2}\right): 13 \mathrm{ml}$ juice, (80\% Jamun: $15 \%$ avocado: $5 \%$ nannari), cane sugar

$\mathrm{T}_{6}\left(\mathrm{~J}_{3} \mathrm{~S}_{1} \mathrm{R}_{2}\right): 16 \mathrm{ml}$ juice, (80\% Jamun: $15 \%$ avocado: $5 \%$ nannari), cane sugar

$\mathrm{T}_{7}\left(\mathrm{~J}_{1} \mathrm{~S}_{2} \mathrm{R}_{1}\right): 10 \mathrm{ml}$ juice, (65\% Jamun: $30 \%$ avocado: $5 \%$ nannari), honey

$\mathrm{T}_{8}\left(\mathrm{~J}_{2} \mathrm{~S}_{2} \mathrm{R}_{1}\right): 13 \mathrm{ml}$ juice, (65\% Jamun: $30 \%$ avocado: $5 \%$ nannari), honey

$\mathrm{T}_{9}\left(\mathrm{~J}_{3} \mathrm{~S}_{2} \mathrm{R}_{1}\right): 16 \mathrm{ml}$ juice, (65\% Jamun: $30 \%$ avocado: $5 \%$ nannari), honey

$\mathrm{T}_{10}\left(\mathrm{~J}_{1} \mathrm{~S}_{2} \mathrm{R}_{2}\right): 10 \mathrm{ml}$ juice, (80\% Jamun: $15 \%$ avocado: $5 \%$ nannari), honey
$\mathrm{T}_{11}\left(\mathrm{~J}_{2} \mathrm{~S}_{2} \mathrm{R}_{2}\right)$ : $13 \mathrm{ml}$ juice, (80\% Jamun: $15 \%$ avocado: $5 \%$ nannari), honey

$\mathrm{T}_{12}\left(\mathrm{~J}_{3} \mathrm{~S}_{2} \mathrm{R}_{2}\right): 16 \mathrm{ml}$ juice, (80\% Jamun: $15 \%$ avocado: $5 \%$ nannari), honey

Various parameters were studied during the evaluation. The fresh pulp was analysed to find out its proximate composition. The $\mathrm{pH}$, titratable acidity, total soluble solids, ascorbic acid, reducing sugar, non-reducing sugar, total sugars, antioxidants activity and anthocyanins were determined and the values were recorded. Similarly, the physico-chemical analysis of jamun blended with avocado and nannari (RTS) were analysed for $\mathrm{pH}$, Titratable acidity, total soluble solids, ascorbic acid, reducing sugar, non-reducing sugar, total sugars, antioxidants activity and anthocyanins during storage at 30 Days intervals for 90 Days at ambient temperature. The data was analyzed by as per the guidelines suggested by Panse and Sukhatne (1978) using complete randomized design.

The proximate chemical analysis of Jamun blended beverages was done for different parameter. The total soluble solids, titrable acidity, total sugars, ascorbic acid and total anthocyanins were estimated as per the procedures outlined by Ranganna, 1986. The total soluble solids was determined by using hand refractometer and expressed in ${ }^{\circ}$ Brix (Erma refractometer), ascorbic acid content was estimated by using 2, 6-dichloro phenol indophenol dye, the total anthocyanins were determined by absorbance of samples at 510 $\mathrm{nm}$ in spectrophotometer. The $\mathrm{pH}$ of RTS was recorded with the help of digital $\mathrm{pH}$ meter.

\section{Results and Discussion}

The results of various RTS recipes prepared using jamun, avocado and nannari either with sugar or honey is presented and discussed for different parameters. An increasing trend was 
observed in total soluble solids content of jamun RTS during storage (Table 2). The maximum TSS of 17.60 per cent was noticed in $\mathrm{T}_{12}[(16 \mathrm{ml}$ juice $),(80$ per cent Jamun, 15 per cent avocado, 5 per cent nannari) and honey]. This increase might be due to hydrolysis of polysaccharides like starch and pectic substances present in the pulp into simpler substances which contributed to increase in TSS content (Ravindra et al., 2012; Smitha et al., 2012; Sudhindra Kumar et al., 2012;Totad et al., 2014 and Priyanka et al., 2015).

The $\mathrm{pH}$ of the prepared beverage recorded an increasing trend during storage. Irrespective of treatment combination and were all nonsignificant maximum $\mathrm{pH}$ of 4.50 was observed in $\mathrm{T}_{1}$ [(10ml juice), (65 per cent Jamun, 30 per cent avocado, 5 per cent nannari) and sugar] (Table 3). A corresponding decrease in acidity might be responsible for it. The acid content in Jamun and nannari may be the reason for its increase in pH (Pooja et al., 2012; Sudhindra Kumar et al., 2012; Totad et al., 2014; Kapoor and Ranote, 2015 and Priyanka et al., 2015).

The acidity of prepared RTS gradually declined during storage corresponding to increase in $\mathrm{pH}$ (Table 3). Maximum reduction
$(0.16 \%)$ was noticed in $\mathrm{T}_{9}$ [(16ml juice), (65 per cent Jamun, 30 per cent avocado, 5 per cent nannari) and honey].

This decrease in acidity might be due to acid hydrolysis of polysaccharides and nonreducing sugars to their simpler components, where acid is utilized for converting them to hexose sugars or complexes in the presence of metal ions (Hamid Salari, 2012 and Pooja et al., 2012; Smitha et al., 2012; Sudhindra Kumar et al., 2012 and Priyanka et al., 2015).

The ascorbic acid content of the RTS beverage recorded decreasing pattern and were all nonsignificant (Table 2). Maximum ascorbic acid content (22.32 mg $100 \mathrm{~g}^{-1}$ ) was recorded in $\mathrm{T}_{12}$ [(16ml juice), (80 per cent Jamun, 15 per cent avocado, 5 per cent nannari) and honey].

Decrease in ascorbic acid content might be due to the effect of storage temperature and catalytic activity of fructose.

Thermal degradation during processing and subsequent oxidation and light reaction were the other possible causes of reduction in ascorbic acid content (Sudhindra Kumar et al., 2012; Totad et al., 2014; Kapoor and Ranote, 2015 and Priyanka et al., 2015).

Table.1 Physical parameters of jamun and avocado fruit

\begin{tabular}{|c|l|c|c|}
\hline Sl. No. & \multicolumn{1}{|c|}{ Parameters } & Jamun & Avocado \\
\hline 1 & Fruit length $(\mathrm{cm})$ & 2.65 & 9.50 \\
\hline 2 & Fruit diameter $(\mathrm{cm})$ & 1.65 & 6.96 \\
\hline 3 & Fruit weight $(\mathrm{g})$ & 20.00 & 152.00 \\
\hline 4 & Pulp (\%) & 74.00 & 66.00 \\
\hline 5 & Seed (\%) & 26.00 & 34.00 \\
\hline
\end{tabular}


Table.2 Effect of Jamun RTS blended with avocado and nannari on TSS ( ${ }^{0}$ Brix) and ascorbic acid content $\left(\mathrm{mg} 100 \mathrm{~g}^{-1}\right)$ during storage

\begin{tabular}{|c|c|c|c|c|c|c|c|c|}
\hline \multirow[t]{2}{*}{ *Treatments } & \multicolumn{4}{|c|}{ TSS ( ${ }^{0}$ Brix) } & \multicolumn{4}{|c|}{ Ascorbic acid (mg $\left.100 \mathrm{~g}^{-1}\right)$} \\
\hline & Fresh & 30 days & 60 days & 90 days & Fresh & 30 days & 60 days & 90 days \\
\hline $\mathrm{T}_{1}\left(\mathrm{~J}_{1} \mathrm{~S}_{1} \mathrm{R}_{1}\right)$ & 15.00 & 15.40 & 16.27 & 17.17 & 22.74 & 21.66 & 20.85 & 20.52 \\
\hline $\mathrm{T}_{2}\left(\mathrm{~J}_{2} \mathrm{~S}_{1} \mathrm{R}_{1}\right)$ & 15.00 & 15.40 & 16.27 & 17.13 & 22.95 & 21.84 & 21.33 & 20.79 \\
\hline $\mathrm{T}_{3}\left(\mathrm{~J}_{3} \mathrm{~S}_{1} \mathrm{R}_{1}\right)$ & 15.00 & 15.47 & 16.23 & 17.17 & 23.16 & 22.23 & 21.42 & 20.85 \\
\hline $\mathrm{T}_{4}\left(\mathrm{~J}_{1} \mathrm{~S}_{1} \mathrm{R}_{2}\right)$ & 15.00 & 15.43 & 16.27 & 17.13 & 23.10 & 21.69 & 21.00 & 20.67 \\
\hline $\mathrm{T}_{5}\left(\mathrm{~J}_{2} \mathrm{~S}_{1} \mathrm{R}_{2}\right)$ & 15.00 & 15.57 & 16.30 & 17.13 & 23.22 & 22.29 & 21.45 & 20.82 \\
\hline $\mathrm{T}_{6}\left(\mathrm{~J}_{3} \mathrm{~S}_{1} \mathrm{R}_{2}\right)$ & 15.00 & 15.50 & 16.33 & 17.23 & 23.37 & 22.62 & 21.75 & 20.88 \\
\hline $\mathrm{T}_{7}\left(\mathrm{~J}_{1} \mathrm{~S}_{2} \mathrm{R}_{1}\right)$ & 15.00 & 15.57 & 16.43 & 17.40 & 23.76 & 22.62 & 21.84 & 21.24 \\
\hline $\mathrm{T}_{8}\left(\mathrm{~J}_{2} \mathrm{~S}_{2} \mathrm{R}_{1}\right)$ & 15.00 & 15.60 & 16.43 & 17.50 & 23.88 & 22.65 & 21.90 & 21.36 \\
\hline $\mathrm{T}_{9}\left(\mathrm{~J}_{3} \mathrm{~S}_{2} \mathrm{R}_{1}\right)$ & 15.00 & 15.63 & 16.37 & 17.53 & 23.97 & 22.71 & 21.87 & 21.45 \\
\hline $\mathrm{T}_{10}\left(\mathrm{~J}_{1} \mathrm{~S}_{2} \mathrm{R}_{2}\right)$ & 15.00 & 15.67 & 16.43 & 17.57 & 24.30 & 23.49 & 22.59 & 22.08 \\
\hline $\mathrm{T}_{11}\left(\mathrm{~J}_{2} \mathrm{~S}_{2} \mathrm{R}_{2}\right)$ & 15.00 & 15.70 & 16.47 & 17.53 & 24.55 & 23.52 & 22.62 & 22.11 \\
\hline $\mathrm{T}_{12}\left(\mathrm{~J}_{3} \mathrm{~S}_{2} \mathrm{R}_{2}\right)$ & 15.00 & 15.73 & 16.43 & 17.60 & 24.72 & 23.94 & 22.77 & 22.32 \\
\hline S. Em \pm & & 0.035 & 0.032 & 0.037 & 0.042 & 0.041 & 0.041 & 0.040 \\
\hline CD @ 0.05 & & 0.101 & 0.093 & 0.109 & NS & NS & NS & NS \\
\hline
\end{tabular}

Note: 1. NS- Non significant, 2. * Treatment details in materials and methods

Table.3 Effect of Jamun RTS blended with avocado and nannari on titratable acidity (\%) and pH content during storage

\begin{tabular}{|c|c|c|c|c|c|c|c|c|}
\hline \multirow[t]{2}{*}{ *Treatments } & \multicolumn{4}{|c|}{ Titratable acidity (\%) } & \multicolumn{4}{|c|}{ pH } \\
\hline & Fresh & 30 days & 60 days & 90 days & Fresh & 30 days & 60 days & 90 days \\
\hline $\mathrm{T}_{1}\left(\mathrm{~J}_{1} \mathrm{~S}_{1} \mathrm{R}_{1}\right)$ & 0.30 & 0.23 & 0.21 & 0.20 & 4.30 & 4.39 & 4.47 & 4.50 \\
\hline $\mathrm{T}_{2}\left(\mathrm{~J}_{2} \mathrm{~S}_{1} \mathrm{R}_{1}\right)$ & 0.30 & 0.22 & 0.20 & 0.19 & 4.25 & 4.31 & 4.39 & 4.45 \\
\hline $\mathrm{T}_{3}\left(\mathrm{~J}_{3} \mathrm{~S}_{1} \mathrm{R}_{1}\right)$ & 0.30 & 0.23 & 0.21 & 0.19 & 4.23 & 4.28 & 4.35 & 4.42 \\
\hline $\mathrm{T}_{4}\left(\mathrm{~J}_{1} \mathrm{~S}_{1} \mathrm{R}_{2}\right)$ & 0.30 & 0.22 & 0.20 & 0.19 & 4.08 & 4.20 & 4.28 & 4.31 \\
\hline $\mathrm{T}_{5}\left(\mathrm{~J}_{2} \mathrm{~S}_{1} \mathrm{R}_{2}\right)$ & 0.30 & 0.22 & 0.20 & 0.18 & 4.00 & 4.14 & 4.26 & 4.27 \\
\hline $\mathrm{T}_{6}\left(\mathrm{~J}_{3} \mathrm{~S}_{1} \mathrm{R}_{2}\right)$ & 0.30 & 0.23 & 0.19 & 0.17 & 3.97 & 4.14 & 4.25 & 4.27 \\
\hline $\mathrm{T}_{7}\left(\mathrm{~J}_{1} \mathrm{~S}_{2} \mathrm{R}_{1}\right)$ & 0.30 & 0.22 & 0.19 & 0.18 & 3.95 & 4.12 & 4.21 & 4.24 \\
\hline $\mathrm{T}_{8}\left(\mathrm{~J}_{2} \mathrm{~S}_{2} \mathrm{R}_{1}\right)$ & 0.30 & 0.22 & 0.19 & 0.17 & 3.95 & 4.12 & 4.21 & 4.23 \\
\hline $\mathrm{T}_{9}\left(\mathrm{~J}_{3} \mathrm{~S}_{2} \mathrm{R}_{1}\right)$ & 0.30 & 0.22 & 0.19 & 0.16 & 3.96 & 4.14 & 4.24 & 4.25 \\
\hline $\mathrm{T}_{10}\left(\mathrm{~J}_{1} \mathrm{~S}_{2} \mathrm{R}_{2}\right)$ & 0.30 & 0.22 & 0.20 & 0.17 & 3.95 & 4.14 & 4.20 & 4.25 \\
\hline $\mathrm{T}_{11}\left(\mathrm{~J}_{2} \mathrm{~S}_{2} \mathrm{R}_{2}\right)$ & 0.30 & 0.22 & 0.20 & 0.18 & 3.94 & 4.16 & 4.21 & 4.28 \\
\hline $\mathrm{T}_{12}\left(\mathrm{~J}_{3} \mathrm{~S}_{2} \mathrm{R}_{2}\right)$ & 0.30 & 0.23 & 0.20 & 0.18 & 3.96 & 4.19 & 4.25 & 4.30 \\
\hline S. Em \pm & & 0.012 & 0.011 & 0.011 & 0.021 & 0.021 & 0.022 & 0.022 \\
\hline CD @ 0.05 & & NS & NS & NS & NS & NS & NS & NS \\
\hline
\end{tabular}

Note: 1. NS- Non significant, 2. * Treatment details in materials and methods 
Table.4 Effect of Jamun RTS blended with avocado and nannari on total anthocyanins (mg $\left.100 \mathrm{~g}^{-1}\right)$ and total antioxidants $(\%)$ content during storage

\begin{tabular}{|c|c|c|c|c|c|c|c|c|}
\hline \multirow[t]{2}{*}{ *Treatments } & \multicolumn{4}{|c|}{ Total anthocyanins $\left(\mathrm{mg} 100 \mathrm{~g}^{-1}\right)$} & \multicolumn{4}{|c|}{ Total antioxidants $(\%)$} \\
\hline & Fresh & 30 days & 60 days & 90 days & Fresh & 30 days & 60 days & 90 days \\
\hline $\mathrm{T}_{1}\left(\mathrm{~J}_{1} \mathrm{~S}_{1} \mathrm{R}_{1}\right)$ & 10.45 & 9.41 & 8.93 & 8.72 & 57.71 & 53.83 & 50.67 & 47.00 \\
\hline $\mathrm{T}_{2}\left(\mathrm{~J}_{2} \mathrm{~S}_{1} \mathrm{R}_{1}\right)$ & 10.57 & 9.74 & 9.37 & 9.24 & 57.83 & 53.67 & 51.33 & 48.33 \\
\hline $\mathrm{T}_{3}\left(\mathrm{~J}_{3} \mathrm{~S}_{1} \mathrm{R}_{1}\right)$ & 10.60 & 10.08 & 9.64 & 9.28 & 59.00 & 55.00 & 51.67 & 49.00 \\
\hline $\mathrm{T}_{4}\left(\mathrm{~J}_{1} \mathrm{~S}_{1} \mathrm{R}_{2}\right)$ & 12.62 & 11.39 & 10.65 & 10.61 & 61.50 & 58.83 & 54.00 & 51.00 \\
\hline $\mathrm{T}_{5}\left(\mathrm{~J}_{2} \mathrm{~S}_{1} \mathrm{R}_{2}\right)$ & 13.04 & 11.93 & 11.68 & 10.65 & 62.83 & 59.00 & 55.00 & 52.00 \\
\hline $\mathrm{T}_{6}\left(\mathrm{~J}_{3} \mathrm{~S}_{1} \mathrm{R}_{2}\right)$ & 13.38 & 12.56 & 12.08 & 11.49 & 63.50 & 60.67 & 56.33 & 53.00 \\
\hline $\mathrm{T}_{7}\left(\mathrm{~J}_{1} \mathrm{~S}_{2} \mathrm{R}_{1}\right)$ & 10.59 & 9.37 & 9.24 & 8.95 & 58.83 & 56.17 & 51.00 & 49.67 \\
\hline $\mathrm{T}_{8}\left(\mathrm{~J}_{2} \mathrm{~S}_{2} \mathrm{R}_{1}\right)$ & 10.61 & 9.71 & 9.53 & 9.47 & 60.67 & 57.00 & 59.00 & 49.67 \\
\hline $\mathrm{T}_{9}\left(\mathrm{~J}_{3} \mathrm{~S}_{2} \mathrm{R}_{1}\right)$ & 10.66 & 10.04 & 9.95 & 9.66 & 61.83 & 58.00 & 53.00 & 51.00 \\
\hline $\mathrm{T}_{10}\left(\mathrm{~J}_{1} \mathrm{~S}_{2} \mathrm{R}_{2}\right)$ & 12.44 & 11.78 & 11.51 & 11.38 & 65.50 & 61.67 & 58.00 & 54.00 \\
\hline $\mathrm{T}_{11}\left(\mathrm{~J}_{2} \mathrm{~S}_{2} \mathrm{R}_{2}\right)$ & 12.70 & 12.26 & 12.05 & 11.70 & 65.83 & 62.67 & 55.34 & 55.00 \\
\hline $\mathrm{T}_{12}\left(\mathrm{~J}_{3} \mathrm{~S}_{2} \mathrm{R}_{2}\right)$ & 12.79 & 12.45 & 12.26 & 11.80 & 66.50 & 63.67 & 60.67 & 56.00 \\
\hline S. Em \pm & 0.031 & 0.139 & 0.092 & 0.0455 & 0.411 & 0.329 & 0.441 & 0.471 \\
\hline CD@ 0.05 & 0.089 & 0.405 & 0.268 & 0.133 & 1.199 & 0.968 & 1.287 & 1.376 \\
\hline
\end{tabular}

Note: 1 . NS- Non significant, 2. * Treatment details in materials and methods

Table.5 Effect of Jamun RTS blended with avocado and nannari on total sugars, reducing sugars and non-reducing sugars during storage

\begin{tabular}{|c|c|c|c|c|c|c|c|c|c|c|c|c|}
\hline \multirow[t]{2}{*}{ *Treatments } & \multicolumn{4}{|c|}{ Total sugars (\%) } & \multicolumn{4}{|c|}{ Reducing sugars (\%) } & \multicolumn{4}{|c|}{ Non-Reducing sugars (\%) } \\
\hline & Fresh & $\begin{array}{c}30 \\
\text { days }\end{array}$ & $\begin{array}{c}60 \\
\text { days }\end{array}$ & $\begin{array}{c}90 \\
\text { days }\end{array}$ & Fresh & $\begin{array}{c}30 \\
\text { days }\end{array}$ & $\begin{array}{c}60 \\
\text { days }\end{array}$ & $\begin{array}{c}90 \\
\text { days }\end{array}$ & Fresh & $\begin{array}{c}30 \\
\text { days }\end{array}$ & $\begin{array}{c}60 \\
\text { days }\end{array}$ & $\begin{array}{c}90 \\
\text { days }\end{array}$ \\
\hline $\mathrm{T}_{1}\left(\mathrm{~J}_{1} \mathrm{~S}_{1} \mathrm{R}_{1}\right)$ & 10.80 & 10.96 & 11.09 & 11.13 & 4.81 & 5.21 & 5.61 & 6.21 & 5.99 & 5.75 & 5.48 & 4.92 \\
\hline $\mathrm{T}_{2}\left(\mathrm{~J}_{2} \mathrm{~S}_{1} \mathrm{R}_{1}\right)$ & 10.87 & 10.98 & 11.14 & 11.25 & 4.71 & 4.96 & 5.42 & 6.13 & 6.16 & 6.02 & 5.72 & 5.12 \\
\hline $\mathrm{T}_{3}\left(\mathrm{~J}_{3} \mathrm{~S}_{1} \mathrm{R}_{1}\right)$ & 11.07 & 11.32 & 11.41 & 11.64 & 4.92 & 5.47 & 5.83 & 6.22 & 6.28 & 5.85 & 5.64 & 5.42 \\
\hline $\mathrm{T}_{4}\left(\mathrm{~J}_{1} \mathrm{~S}_{1} \mathrm{R}_{2}\right)$ & 11.21 & 11.54 & 11.68 & 11.87 & 5.53 & 5.87 & 6.13 & 6.42 & 5.88 & 5.67 & 5.54 & 5.45 \\
\hline $\mathrm{T}_{5}\left(\mathrm{~J}_{2} \mathrm{~S}_{1} \mathrm{R}_{2}\right)$ & 11.26 & 11.69 & 11.76 & 11.86 & 5.61 & 5.97 & 6.22 & 6.52 & 5.88 & 5.72 & 5.52 & 5.10 \\
\hline $\mathrm{T}_{6}\left(\mathrm{~J}_{3} \mathrm{~S}_{1} \mathrm{R}_{2}\right)$ & 11.41 & 11.81 & 11.97 & 12.07 & 5.82 & 6.21 & 6.36 & 6.57 & 5.89 & 5.60 & 5.61 & 5.50 \\
\hline $\mathrm{T}_{7}\left(\mathrm{~J}_{1} \mathrm{~S}_{2} \mathrm{R}_{1}\right)$ & 11.67 & 12.22 & 12.33 & 12.43 & 6.72 & 6.90 & 7.25 & 7.42 & 5.39 & 5.32 & 5.08 & 5.01 \\
\hline $\mathrm{T}_{8}\left(\mathrm{~J}_{2} \mathrm{~S}_{2} \mathrm{R}_{1}\right)$ & 11.77 & 12.44 & 12.53 & 12.65 & 6.72 & 6.92 & 7.25 & 7.54 & 5.54 & 5.52 & 5.28 & 5.11 \\
\hline $\mathrm{T}_{9}\left(\mathrm{~J}_{3} \mathrm{~S}_{2} \mathrm{R}_{1}\right)$ & 11.82 & 12.42 & 12.58 & 12.73 & 6.76 & 6.96 & 7.29 & 7.50 & 5.55 & 5.46 & 5.29 & 5.22 \\
\hline $\mathrm{T}_{10}\left(\mathrm{~J}_{1} \mathrm{~S}_{2} \mathrm{R}_{2}\right)$ & 12.01 & 12.68 & 12.83 & 12.93 & 7.27 & 7.43 & 7.81 & 7.93 & 5.34 & 5.25 & 5.02 & 5.00 \\
\hline $\mathrm{T}_{11}\left(\mathrm{~J}_{2} \mathrm{~S}_{2} \mathrm{R}_{2}\right)$ & 12.07 & 13.02 & 13.17 & 13.22 & 7.46 & 7.62 & 7.82 & 8.18 & 5.23 & 5.40 & 5.35 & 5.04 \\
\hline $\mathrm{T}_{12}\left(\mathrm{~J}_{3} \mathrm{~S}_{2} \mathrm{R}_{2}\right)$ & 12.28 & 13.15 & 13.26 & 13.42 & 7.67 & 7.72 & 7.84 & 8.30 & 5.34 & 5.44 & 5.43 & 5.13 \\
\hline S. Em \pm & 0.011 & 0.011 & 0.008 & 0.010 & 0.010 & 0.015 & 0.013 & 0.011 & 0.011 & 0.022 & 0.016 & 0.014 \\
\hline CD@0.05 & NS & 0.032 & 0.026 & 0.030 & 0.030 & 0.044 & 0.038 & NS & 0.032 & 0.065 & 0.047 & NS \\
\hline
\end{tabular}

Note: 1. NS- Non significant, 2. * Treatment details in materials and methods

The anthocyanin content of blended RTS decreased during storage. Maximum retention $\left(11.8 \mathrm{mg} 100 \mathrm{ml}^{-1}\right)$ of anthocyanin content was noticed in $\mathrm{T}_{12}$ [(16ml juice), (80 per cent Jamun, 15 per cent avocado, 5 per cent nannari) and honey] (Table 4). The decrease may be due to thermal degradation during processing and oxidation process occurring during storage as anthocyanin are photosensitive in nature or due to nonenzymatic browning reactions (Kapoor and Ranote, 2015 and Priyanka et al., 2015). 
The antioxidant content of blended RTS decreased during storage (Table 4). Maximum inhibition $(56.00 \%)$ of total antioxidant was observed in $\mathrm{T}_{12}$ [(16ml juice), (80 per cent Jamun, 15 per cent avocado, 5 per cent nannari) and honey]. Decrease in the antioxidative property may be due to degradation in anthocyanin, ascorbic acid, total phenols, change in the $\mathrm{pH}$ composition, which are responsible for stability of antioxidant properties. Similar observations were recorded by Priyanka et al., (2015), Kapoor and Ranote (2015).

The total sugar content of Jamun blended RTS increased during storage (Table 5). Maximum of 13.42 per cent of total sugars was found in $\mathrm{T}_{12}$ [(16ml juice), (80 per cent Jamun, 15 per cent avocado, 5 per cent nannari) and honey], maximum reduction of 8.30 per cent in reducing sugar was found in $\mathrm{T}_{12}$ [(16ml juice), (80 per cent Jamun, 15 per cent avocado, 5 per cent nannari) and honey] and 5.50 percent of non-reducing sugar was observed in $\mathrm{T}_{6}$ [(16ml juice), ( 80 per cent Jamun, 15 per cent avocado, 5 per cent nannari) and sugar]. It could be attributed to the acid hydrolysis of polysaccharides, which resulted in increase in soluble sugars content and high composition of juice in the treatment. Similar observations were recorded by Pooja et al., (2012), Totad et al., (2014) and Priyanka et al., (2015).

\section{References}

Anonymous, 1990. The Wealth of India. Raw materials, 5(1): 92-98.

Chadha, K. L. 2001. Horticulture industry in India. In: Handbook of Horticulture. Ed. Chadha, K. L. Directorate of information and publication of Agriculture, Indian Council of Agric. Res., New Delhi, Pp. 4-7.

Hamid Salari, Sreenivas, K. N., Krishna, H.C., Shankarappa, T.H. and
Ramakrishna, $\quad$ B.M. 2012.

Standardization of recipe for muskmelon blended beverages. $J$. Interacad. 16 (4): 878-886.

Kapoor, S. and Ranote, P. S. 2015. Antioxidant potentials and quality of blended pear-Jamun (Syzygium cuminii L.) juice. Int. Res. J. Biol. Sci., 4(4): 3037.

Nayar, R. C., Pattanshetty, J. K., Mary, Z. and Yoganarasimhan, S. N. 1978. Pharmacognostical studies on the root of Decalepis hamiltonii Wt \& Arn. and comparison with Hemidesmus indicus (L.) R.Br. Proceedings of the Indian Academy Sci., 87(2): 37-48.

Panse and Sukhatne, 1978. Statistical methods for agricultural workers. Indian Council of Agric. Res., New Delhi, 78pp.

Pooja, B., Sreenivas, K. N. and Sreeramu, B. S. 2012. Development of cost-effective palatable and shelf-stable blended beverage of pummelo (Citrus grandis Linn.) Fruits, 67: 249-256.

Priyanka, N., Dorajeerao, A. V. D., Sudhavani, V. and Umakrishna, K. 2015. Physico-chemical characters and sensory evaluation of Jamun based blended beverages during storage. $\mathrm{Pl}$. Archives, 15(2): 939-946.

Ranganna, 1986. Handbook of analysis and quality control for fruit and vegetable products. II $^{\text {nd }}$ edition. Tata McGrawHill Publishing Company Ltd., New Delhi, India, 1112pp.

Ravindra, V., Sreenivas, K.N. and Shankarappa, T.H. 2012. Value addition and technology development: Shatavari, aloe and mango ginger blended nectar beverage. Envi. Ecol. 30 (3): 495-500.

Smitha, N. K., Sreeramu, B. S., Shrinivas, S., Chikkur, A. S., Parmeshwar and Kantharaj, Y. 2012. Standardization of processing technology and sensory evaluation of avocado squash blended 
with sapota and aloe. Int. J. Proc. Postharvest Technol., 3(1): 94-97.

Srivastava, R. P. and Kumar, S. 1998. In: Fruit and Vegetable Preservation Principles and Practices. International Book Distributing Co., Lucknow, 6498.

Sudhindra Kumar, S. N., Sreenivas, K. N., Shankarappa T. H. and Ravindra, V. 2012. Standardization of recipe for value added nutraceutical beverages of guava blended with Aloe vera and roselle, Envi. Ecol. 30 (3B): 995-1001.

Swamy, S. B., Thakor, N. J., Haldankar, P. M. and Patil, M. M. 2012. Processing and value addition in Jamun. Int. J. Proc. Postharvest Technol., 3(1): 147-149.

Totad, B. M., Ramakrishna, M. and Sreenivas, K. N. 2014. Development of value added products from sapota (Manilkara achrus) blended with jackfruit and avocado. Int. J. Proc. Postharvest Technol., 5(1): 48-53.

\section{How to cite this article:}

Arshad Khayum, H.C. Krishna, A.P. Mallikarjuna Gowda, G.K. Sadananda and Shankarappa, T.H. 2018. Formulation and Evaluation of Jamun RTS Blended with Avocado and Nannari - A Nutraceutical Drink. Int.J.Curr.Microbiol.App.Sci. 7(06): 535-542.

doi: https://doi.org/10.20546/ijcmas.2018.706.061 